\title{
COMPARAÇÃO MORFOFISIOLÓGICA DE BIÓTIPOS DE MILHÃ SOB DIFERENTES CONDIÇÕES DE IRRIGAÇÃO
}

\author{
Lisiane Camponogara Fontana ${ }^{1}$, Karen Piraine Martins ${ }^{2}$, Lais Tessari Perboni ${ }^{3}$, Ana Claudia \\ Langaro $^{2}$, Claudia de Oliveira ${ }^{3}$, Dirceu Agostinetto ${ }^{4}$ \\ ${ }^{1}$ Doutora em Fitossanidade - Universidade Federal de Pelotas. \\ ${ }^{2}$ Mestre em Fitossanidade - Universidade Federal de Pelotas. \\ ${ }^{3}$ Doutoranda em Fitossanidade - Universidade Federal de Pelotas. \\ ${ }^{4}$ Professor Associado - Universidade Federal de Pelotas.
}

RESUMO: As espécies do gênero Digitaria (milhã) adaptam-se a diferentes ambientes e possuem elevado potencial competitivo o que causa perdas de produtividade em cultivos agrícolas. No Rio Grande do Sul (RS) encontram-se, principalmente, em culturas de sequeiro como milho e soja, entretanto, essa planta daninha tem sido frequentemente encontrada em lavouras de arroz irrigado. Em vista do exposto, o objetivo do trabalho foi avaliar as respostas morfológicas de diferentes biótipos de milhã quanto à variação dos teores de umidade do solo. O experimento foi conduzido em casa de vegetação no ano agrícola 2010/11, em delineamento experimental completamente casualizado, em esquema fatorial, com três repetições. Foram testados 19 biótipos de milhã, coletados em diferentes regiões do RS; e, três condições de irrigação (seco, inundação com duas folhas ou com um afilho). As variáveis avaliadas foram: teor de clorofila, área foliar e massa da matéria seca da parte aérea. Os resultados permitem concluir que os biótipos de milhã diferem quanto a adaptação ao ambiente de desenvolvimento e que a condição de sequeiro favorece a espécie, comparativamente a inundação.

Palavras-chave: D. sanguinalis. D. ciliaris. D. aequiglumis. D. bicornis. Plantas daninhas.

\section{MORPHOLOGICAL ANALYSIS OF CRABGRASS BIOTYPES DEVELOPED IN DIFFERENT CONDITIONS OF FLOOD}

\begin{abstract}
Digitaria is a species that adapts to different environments, mainly by having high competitive potential. Why be a weed, cause significant yield losses when present in agricultural crops. In Rio Grande do Sul (RS) is found primarily in crops such as corn, soybeans and sorghum. However, this weed has been frequently found in irrigated rice fields. The aim of this paper was to evaluate the morphological responses of different biotypes of Digitaria as to changes in the levels of soil moisture under controlled environmental conditions. The experiment was conducted in a greenhouse in the growing season 2010/11 The experimental design was completely randomized in factorial with three replications. Where tested 19 biotypes of Digitaria, collected in different regions of the state of Rio Grande do Sul, the flood of the soil in two stages of plant growth (two leaves and one tiller) and a control in the dry condition. The variables evaluated were content of chlorophyll, leaf area and dry matter of aerial part. The results indicate that the crabgrass biotypes differ adaptation
\end{abstract}

Cultura Agronômica, Ilha Solteira, v.24, n.3, p.319-326, 2015 
to the development environment and that promotes an upland species, compared to flood.

Key words: D. sanguinalis. D. ciliaris. D. aequiglumis. D. bicornis. Weeds.

\section{INTRODUÇÃO}

O arroz (Oryza sativa L.) é o principal alimento para grande parte a população mundial e, juntamente com milho e trigo, constitui na maioria dos cereais produzidos no mundo (FAO, 2014). Rio Grande do Sul e Santa Catarina são os principais produtores sendo responsáveis por $74 \%$ da produção brasileira na última safra. Entretanto, mesmo com produtividade média relativamente crescente, ainda existem fatores que limitam o seu crescimento, como a ocorrência de plantas daninhas, as quais constituem um dos principais entraves para que a cultura atinja o potencial máximo de produtividade.

O sistema de cultivo do arroz irrigado é fundamentalmente conduzido sob irrigação por inundação, que consta na permanência de lâmina de água sobre a superfície do solo durante praticamente todo o ciclo da cultura (SOSBAI, 2011). A lâmina de água reduz o crescimento e desenvolvimento de diversas plantas daninhas funcionando como barreira física, devido à redução da disponibilidade de oxigênio (BORRELL et al., 1997; VILLA et al., 2006).

As espécies pertencentes ao gênero Digitaria, conhecidas popularmente por milhã, pertencem a família poaceae, de hábito de crescimento herbáceo, com crescimento inicial lento, folhas alternadas dísticas, lanceoladas e paralelinérveas, com reprodução via semente (cariopse) com grande potencial de dispersão (KISSMANN; GROTH, 2007). Adapta-se a diferentes ambientes, possuindo elevado potencial competitivo, causando perdas de produtividade em diversas culturas.

No Brasil a espécie é considerada como uma das mais agressivas da agricultura (LÓPEZ OVEJERO et al., 2007), infestante tanto durante a primavera/verão quanto no outono/inverno (JAKELAITIS et al. 2010). No Rio Grande do Sul infesta principalmente culturas de sequeiro como milho, soja e sorgo, entretanto, tem sido encontrada em lavouras de arroz irrigado devido, possivelmente, a sua facilidade de adaptação.

A capacidade das espécies de se mostrarem adaptadas a períodos de encharcamento ou inundação do solo pode ser atribuída a mecanismos de adaptação morfo-anatômicos, fisiológicos e bioquímicos (ISHIDA et al., 2002). Dentre as espécies tolerantes ao alagamento, algumas crescem e se desenvolvem melhor sob inundação e apresentam, por exemplo, alterações morfoanatômicas que auxiliam na aeração interna da planta (MEDRI et al., 2002); outras investem menos no crescimento, reduzindo o consumo de energia, retomando o crescimento após o estresse (MEDRI et al. 2002).

Dependendo da espécie, da velocidade de encharcamento do solo, da altura da lâmina d'água e do tempo de submersão, esses mecanismos podem ser mais evidentes, favorecendo a sobrevivência das plantas nestes ambientes (ISHIDA et al., 2002). O excesso de água diminui a difusão de gases reduzindo a disponibilidade de oxigênio no solo e, consequentemente, para o sistema radicular das plantas. Essa depleção na disponibilidade de 
oxigênio sinaliza para um estresse abiótico fazendo, desta forma, com que a planta altere o seu metabolismo normal (DAT et al., 2004).

Em estádio mais avançado, esses danos podem ser exteriorizados pela redução nos teores de clorofilas resultantes da degradação e/ou decréscimo de sua síntese como contatado em folhas de outras espécies submetidas a diferentes níveis de inundação (HUANG et al., 1994; PEZESHKI et al., 1996). Como resultado imediato o crescimento é reduzido, podendo chegar a níveis de danos que não permitem a retomada do crescimento após a drenagem.

O trabalho teve por objetivo avaliar as respostas morfofisiológicas de diferentes biótipos de milhã (Digitaria spp.) quanto à variação dos teores de umidade em condições ambientais controladas.

\section{MATERIAL E MÉTODOS}

O experimento foi desenvolvido em casa de vegetação no ano agrícola 2010/11, em delineamento experimental completamente casualizado, com três repetições. As unidades experimentais foram compostas por vasos plásticos com capacidade volumétrica de oito litros, preenchidos com solo peneirado, oriundo de área de várzea, classificado como Planossolo Hidromórfico eutrófico solódico, pertencente à Unidade de Mapeamento Pelotas (EMBRAPA, 1999). A correção da fertilidade do solo foi realizada conforme as recomendações técnicas para a cultura do arroz irrigado (SOSBAI, 2011). Os biótipos foram semeados em densidades superiores as desejadas, com posterior desbaste para a retirada do excesso de plantas mantendo-se população de dez plantas por vaso.

Os tratamentos foram arranjados em esquema fatorial sendo o fator A composto por três condições de irrigação (seco, inundação com duas folhas ou com um afilho) e o fator B por biótipos de Digitaria spp., coletados em diferentes regiões do Rio Grande do Sul e pertencentes a diferentes espécies (Tabela 1).

As variáveis avaliadas foram: teor de clorofila (CL), área foliar (AF) e massa da matéria seca da parte aérea (MMSPA), aos 49 dias após a aplicação dos tratamentos. O CL foi determinado em dois pontos da última folha completamente desenvolvida (base e ápice da folha), com auxílio de medidor portátil de clorofila (modelo SPAD); a AF foi quantificada com medidor de área foliar, Modelo 3100C; e, a MMSPA foi obtida pela secagem do material em estufa, a temperatura de $60^{\circ} \mathrm{C}$, até atingir peso constante.

Os dados foram analisados quanto à normalidade, pelo teste de Shapiro-Wilk e à homocedasticidade, pelo teste de Hartley, sendo posteriormente submetidos à análise de variância $(\mathrm{p} \leq 0,05)$. No caso de constatação de significância estatística para os biótipos e para as condições de inundação, foi realizada a comparação entre as médias pelo teste de Duncan $(\mathrm{p} \leq 0,05)$. 
Tabela 1. Identificação botânica e localização geográfica de acessos de milhã (Digitaria) coletados em áreas agrícolas de diferentes municípios do RS, 2009.

\begin{tabular}{|c|c|c|c|c|c|}
\hline Município & Acesso & Espécie & Latitude & Longitude & $\begin{array}{c}\text { Altitude } \\
\text { (m) }\end{array}$ \\
\hline Cachoeira do Sul ${ }^{2}$ & 6 & D. ciliaris & -30.200864 & -52.920639 & 46 \\
\hline São Sepé ${ }^{2}$ & 12 & D. ciliaris & -29.966910 & -53.696307 & 53 \\
\hline Itaqui ${ }^{2}$ & 24 & D. ciliaris & -29.340734 & -56.633031 & 64 \\
\hline \multirow[t]{2}{*}{ Uruguaiana $^{2}$} & 34 & D. sanguinalis & -29.798608 & -56.982144 & 70 \\
\hline & 35 & D. ciliaris & -29.796283 & -56.981064 & 77 \\
\hline Rosário do Sul ${ }^{2}$ & 38 & D. ciliaris & -30.283720 & -54.892779 & 97 \\
\hline \multirow[t]{2}{*}{ Dom Pedrito $^{2}$} & 46 & D. sanguinalis & -30.797976 & -55.002466 & 108 \\
\hline & 47 & D. ciliaris & -30.798134 & -55.002518 & 110 \\
\hline Arroio Grande ${ }^{2}$ & 63 & D. sanguinalis & -32.238315 & -53.043355 & 33 \\
\hline Camaquã $^{2}$ & 66 & D. ciliaris & -30.903028 & -51.703441 & 29 \\
\hline Tapes ${ }^{2}$ & 73 & D. ciliaris & -30.657912 & -51.460523 & 14 \\
\hline \multirow[t]{3}{*}{ S. Vitória do Palmar ${ }^{2}$} & 83 & D. aequiglumis & -32.909343 & -52.697922 & 6 \\
\hline & 85 & D. sanguinalis & -32.901371 & -52.725506 & 13 \\
\hline & 87 & D. ciliaris & -32.901353 & -52.725523 & 13 \\
\hline \multirow[t]{2}{*}{ Mostardas } & 101 & D. ciliaris & -30.503781 & -50.563941 & 10 \\
\hline & 106 & D. sanguinalis & -30.492992 & -50.547473 & 1 \\
\hline \multirow[t]{2}{*}{ Passo Fundo ${ }^{3}$} & 118 & D. bicornis & -28.248022 & -52.276402 & 733 \\
\hline & 119 & D. ciliaris & -28.248057 & -52.276350 & 733 \\
\hline Cruz Alta ${ }^{3}$ & 148 & D. ciliaris & -28.603864 & -53.674554 & 411 \\
\hline
\end{tabular}

${ }^{1}$ Número da exsicata, com duplicata, depositada no herbário PEL (UFPEL); ${ }^{2}$ Coletados em lavouras de arroz irrigado; ${ }^{3}$ Coletados em lavouras de soja.

\section{RESULTADOS E DISCUSSÃO}

Não foi observada interação entre os fatores biótipos e estresse por inundação. Quanto as épocas de entrada de água, houve diferença estatística para todas as variáveis analisadas (Tabela 2). Já, entre biótipos não se observou diferença para a variável CL, entretanto para as variáveis AF e MMSPA, os biótipos diferiram entre si (Tabela 3).

Para a variável CL, a condição sem inundação apresentou maior valor quando comparado com inundado com duas folhas ou um afilho (Tabela 2). Resultados da literatura corroboram com os observados, onde se verificou diminuição no conteúdo de CL após 10 dias de inundação em plântulas de Eryithrina variegata L (MUTHUCHELIAN et al., 1996). Também Hurng e Kao (1993) observaram que os efeitos da inundação induziram a menores quantidades de CL em folhas de plantas de tabaco, comparativamente ao tratamento controle. Essa redução pode ser consequência do acúmulo de etileno (SENA GOMES e KOZLOWSKI, 1998) e/ou redução na síntese de citocininas (ZHANG et al., 2000).

Cultura Agronômica, Ilha Solteira, v.24, n.3, p.319-326, 2015 
Tabela 2. Diferenças relativas para as variáveis teores de clorofila (CL), área foliar (AF) e massa da matéria seca da parte aérea (MMSPA) de Digitaria spp., aos 49 dias após a aplicação dos tratamentos. Capão do Leão- RS, 2010/11.

\begin{tabular}{llrc}
\hline Alagamento & \multicolumn{1}{c}{$\mathbf{C L} \stackrel{\mathbf{l}}{\mathbf{A F}\left(\mathbf{c m}^{2}\right)}$} & $\mathbf{M M S P A}(\mathbf{g})$ \\
\hline Sem inundação & $33,69 \mathrm{a}^{\underline{2}}$ & $198,50 \mathrm{a}$ & $0,99 \mathrm{a}$ \\
Inundado com duas folhas & $25,38 \mathrm{~b}$ & $98,61 \mathrm{c}$ & $0,54 \mathrm{c}$ \\
Inundado com um afilho & $24,91 \mathrm{~b}$ & $135,33 \mathrm{~b}$ & $0,80 \mathrm{~b}$ \\
\hline
\end{tabular}

1] Leitura de clorofilômetro (leitura SPAD)

2/ Médias seguidas por letra diferente na coluna diferem entre si pelo teste de Duncan $(p \leq 0,05)$.

Para as variáveis AF e MMSPA a condição sem irrigação por inundação apresentou maiores valores quando comparada com as demais, sendo que a condição de inundação com um afilho teve maior AF e MMSPA que à condição de alagamento com duas folhas (Tabela 2). Em plantas de Setaria anceps o crescimento das folhas também foi reduzido após cinco dias da imposição do estresse sob condições de completa inundação, a taxa de crescimento foliar nessa espécie foi reduzida em mais de 50\%, comparativamente a taxa média obtida nas plantas controle (ISHIDA, 2002). Para muitas espécies, a redução da massa seca da parte aérea correlaciona-se com a morte e/ou com a inibição do crescimento de raízes em resposta à deficiência de oxigênio no solo (PEZESHKI e DELAUNE, 1990; SINGH et al., 1991; MEDRI et al., 1998).

Os resultados dessa pesquisa corroboram com os resultados constatados por Huang et al. (1994) e Joseph e Yelenosk (1991), ao estudarem respectivamente plantas de aveia e de citrus em condições de solos inundados. Esses autores sugeriram que a redução nos teores de clorofila interferiu diretamente nas taxas fotossintéticas, reduzindo o crescimento foliar e a produção de massa da matéria seca. Ainda, Pezeshki et al. (1996) também observaram resultados semelhantes ao estudarem espécies arbóreas em condição de solo encharcado.

Os casos citados da literatura evidenciam que a submersão das plantas por longos períodos de tempo afetam o aparato fotossintético e fotoquímico, reduzem o crescimento de folhas, colmos e raízes, podendo resultar na morte das plantas (KOZLOWSKI, 1984; SCHAFFER et al., 1992; SCHAFFER, 1998). Cabe ressaltar que nos presente estudo não houve morte de plantas o que confirma a capacidade adaptativa dos biótipos de Digitaria a imposição do estresse sob condições e períodos de inundação.

Na comparação entre biótipos, para a variável AF, observou-se que o biótipo 85 (Ijuí) apresentou maior valor absoluto, diferindo estatisticamente dos biótipos 38 (Rosário do Sul), 73 (Tapes) e 119 (Passo Fundo), porém não diferiu dos demais biótipos (Tabela 3).

Para a variável MMSPA, houve diferença entre os biótipos testados, onde o biótipo 106 (Caçapava do Sul) apresentou maior valor absoluto, diferindo estatisticamente dos biótipos 24 (Itaqui), 34 (Uruguaiana), 35 (Uruguaiana), 38 (Rosário do Sul), 63 (Arroio Grande) e 119 (Passo Fundo), não diferindo dos demais. 
Tabela 3. Diferenças relativas para as variáveis área foliar (AF) e massa da matéria seca da parte aérea (MMSPA) dos biótipos de Digitaria spp. aos 49 dias após a aplicação dos tratamentos. Capão do Leão-RS, 2010/11.

\begin{tabular}{cll}
\hline Biótipos & AF $\left(\mathbf{c m}^{\mathbf{2}}\right)$ & MMSPA (g) \\
\hline 6 & $182,58 \mathrm{ab}^{1 /}$ & $0,94 \mathrm{abc}$ \\
12 & $159,51 \mathrm{abc}$ & $0,83 \mathrm{abc}$ \\
18 & $142,97 \mathrm{abc}$ & $0,87 \mathrm{abc}$ \\
24 & $131,21 \mathrm{abc}$ & $0,69 \mathrm{bc}$ \\
34 & $142,62 \mathrm{abc}$ & $0,69 \mathrm{bc}$ \\
35 & $131,71 \mathrm{abc}$ & $0,68 \mathrm{bc}$ \\
38 & $107,18 \mathrm{c}$ & $0,59 \mathrm{c}$ \\
46 & $155,18 \mathrm{abc}$ & $0,78 \mathrm{abc}$ \\
47 & $128,67 \mathrm{abc}$ & $0,75 \mathrm{abc}$ \\
63 & $153,43 \mathrm{abc}$ & $0,60 \mathrm{c}$ \\
66 & $139,13 \mathrm{abc}$ & $0,76 \mathrm{abc}$ \\
73 & $122,78 \mathrm{bc}$ & $0,77 \mathrm{abc}$ \\
83 & $138,81 \mathrm{abc}$ & $0,83 \mathrm{abc}$ \\
85 & $190,16 \mathrm{a}$ & $0,96 \mathrm{ab}$ \\
87 & $149,12 \mathrm{abc}$ & $0,87 \mathrm{abc}$ \\
101 & $135,23 \mathrm{abc}$ & $0,77 \mathrm{abc}$ \\
106 & $184,34 \mathrm{ab}$ & $1,05 \mathrm{a}$ \\
118 & $129,79 \mathrm{abc}$ & $0,77 \mathrm{abc}$ \\
119 & $114,48 \mathrm{c}$ & $0,65 \mathrm{bc}$ \\
\hline
\end{tabular}

Médias seguidas por letra diferente na coluna diferem entre si pelo teste de Duncan $(\mathrm{p} \leq 0,05)$.

\section{CONCLUSÃO}

Os biótipos de milhã diferem quanto à adaptação ao ambiente de desenvolvimento em que a condição de sequeiro favorece a espécie, comparativamente a inundação.

\section{REFERÊNCIAS BIBLIOGRÁFICAS}

BORRELL, A; GARSIDEA, A.; FUKAI, S. Improving efficiency of water use for irrigated rice in a semi-arid tropical environment. Field Crops Research, Bonn, v. 52, n. 3, p.231-248, 1997.

DAT, J. F.; CAPELli, N.; FOLZER, H.; BOURGEADE, P.; BADOT, P. M. Sensing and signaling during plant flooding. Plant Physiology and Biochemistry, Bari, v. 42, n. 4, p.273$282,2004$.

EMPRESA BRASILEIRA DE PESQUISA AGROPECUÁRIA - EMBRAPA. Centro Nacional de Pesquisa Agropecuária de Solos (Rio de Janeiro, RJ). Sistema brasileiro de 
classificação de solos. Brasília: Embrapa Produção de Informação / Rio de Janeiro: Embrapa Solos, 1999. 412 p.

FAO. Food and Agriculture Organization of the United Nations. Production of cereals and share in world. 2014. Disponível em: <http://faostat.fao.org/site/339/default.aspx>. Acesso em: 15 jan. 2014.

HUANG, B.; JOHNSON, J. H.; NESMITH, S.; BRIDGES, D. C. Growth, physiological and anatomical responses of two wheat genotypes to waterlogging and nutrient supply. Journal of Experimental Botany, Lancaster, v. 45, n. 2, p.193-202. 1994.

HURNG, W. P; KAO, C. G. H. Loss of starch andincrease alpha - amylase activity in leaves of flooded tobacco plants. Plant Cell Physiology, Sendai, v. 34, n. 4, p.531-534, 1993.

ISHIDA, F. Y; OLIVEIRA, L. E. M.; CARVALHO, C. J. R.; ALVES. J. D. Efeitos da inundação parcial e total sobre o crescimento, teor de CL e fluorescência de Setaria anceps e Paspalum repens. Ciência e Agrotecnologia, Lavras, v. 26, n. 6, p.1152-1159, 2002.

JAKELAITIS, A.; GIL, J. O.; SIMÕES, L.P.; SOUZA, K.V.; LUDTKE, J. Efeitos da interferência de plantas daninhas na implantação de pastagem de Brachiaria brizantha. Revista Caatinga, Mossoró, v. 23, n. 1, p.8-14, 2010.

JOSEPH, C. V. V.; YELENOSK, Y. Photosyntethic responses of citrus trees to soil flooding. Physiologia Plantarum, Malden, v. 81, n. 1, p.7-14, 1991.

KISSMANN, K. G.; GROTH, D. Plantas infestantes e nocivas, 1. ed. São Paulo: BASF, Tomo I, 2007. 824 p.

KOZLOWSKI, T. T. Responses of woody plants to flooding. In: KOZLOWSKI, T. T. (Ed.). Flooding and plant growth. Orlando: Academic Press, 1984. cap. 4, p. 129-163.

LÓPEZ OVEJERO, R. F.; NOVO, M.C.S.S.; CARVALHO, S.J.P.; NICOLAI, M.; CHRISTOFFOLETI, P.J. Crescimento e competitividade de biótipos de capim-colchão resistente e suscetível aos herbicidas inibidores da Acetil Coenzima A Carboxilase. Pesquisa Agropecuária Brasileira, Brasília, v. 42, n. 1, p.1-8, 2007.

MEDRI, M. E.; BIANCHINI, E.; PIMENTA, J. A.; COLLI, S.; MULLER, C. Estudos sobre a tolerância ao alagamento em espécies arbóreas nativas da bacia do rio Tibagi. In: MEDRI, M. E.; BIANCHINI, E.; SHIBATTA, O. A.; PIMENTA, J. A. (Ed.). A bacia do Rio Tibagi. Londrina: Edição dos editores, 2002. p. 133-172.

MEDRI, M. E.; BIANCHINI, E.; PIMENTA, J. A.; DELGADO, M. F.; CORREA, G. T. Aspectos morfo-anatômicos e fisiológicos de Peltophorum dubium (Spr.) Taub. submetida ao

Cultura Agronômica, Ilha Solteira, v.24, n.3, p.319-326, 2015 
alagamento e à aplicação de etrel. Brazilian Journal of Botany, São Paulo, v. 21, n. 3, p.261267, 1998.

MUTHUCHELIAN, K.; MURUGAN, C.; HARIGOVINDAN, R.; NEDUNCHEZHIAN, N.; KULANDAIVELU, G. Ameliorating effect of triacontanol on salt stressed Erythrina variegata seedlings. Changes in growth, biomass, pigments and solute accumulation. Biologia Plantarum, Prague, v. 38, n. 1, p.133-136, 1996.

PEZESHKI, S.R.; DELAUNE, R.D. Influence of sediment oxidation-reduction potencial on root elongation in Spartina patens. Acta Oecologica, Fribourg, v. 11, n. 3, p. 377-383, 1990.

PEZESHKI, S. R., PARDUE, J. H.; DeLAUNE, R. D. Leaf gas exchange and growth of floodtolerant and flood- sensitive tree species under low soil redox conditions. Tree Physiology, Victoria, v. 16, p.453-458, 1996.

SCHAFFER, B. Flooding responses and water-use efficiency of subtropical fruit trees in an environmentally sensitive Wetland. Annals of Botany, Exeter, v. 81, n. 4, p.475-481, 1998.

SCHAFFER, B.; ANDERSON, P. C.; PLOETZ, R. C. Responses of fruit crops to flooding. In: JANICK, J. Horticultural Reviews, Oxford: John Wiley \& Sons, Inc., v. 13, 1992. cap. 7, p. 257-313.

SENA GOMES, A. R.; KOZLOWSKI, T. T. Physiological and growth responses to flooding of seedlings of Hevea brasiliensis. Biotropica, Lawrence, v. 20, n. 4, p. 286-296, 1988.

SINGH, B. P.; TUCKER, K. A.; SUTTON, J. D.; BHARDWAJ, H. L. Flooding reduces gas exchange and growth in snap bean. HortScience, Washington, v. 26, n. 4, p. 372-373, 1991.

SOCIEDADE SUL BRASILEIRA DE ARROZ IRRIGADO - SOSBAI. Arroz irrigado: recomendações Técnicas da Pesquisa para o Sul do Brasil. In: REUNIÃO TÉCNICA DA CULTURA DO ARROZ IRRIGADO, 7, Balneário Camburiú, 2011. Anais... Balneário Camburiú: Sosbai, 2011. p. 188.

VILLA, S. C. C.; MARCHEZAN, E.; AVILA, L. A.; MASSONI, P. F. S.; TELO, G. M.; MACHADO, S. L. O.; CAMARGO, E. R. Arroz tolerante a imidazolinonas: controle do arroz-vermelho, fluxo gênico e efeito residual do herbicida em culturas sucessoras nãotolerantes. Planta Daninha, Viçosa, v.24, n. 4, p.761-768, 2006.

ZHANG, J.; VAN TOAI, T.; HUYNH, L.; PREISZNER, J. Development of flooding-tolerant Arabidopsis thaliana by autoregulated cytokinin production. Molecular Breeding, Lleida, v. 6, p. 135-144, 2000. 